Published in final edited form as:

Pediatr Crit Care Med. 2016 June ; 17(6): 522-530. doi:10.1097/PCC.0000000000000760.

\title{
Comparison of Pediatric Severe Sepsis Managed in U.S. and European ICUs
}

John S. Giuliano Jr, MD¹, Barry P. Markovitz, MD, MPH², Joe Brierley, MD ${ }^{3}$, Richard Levin, MD $^{4}$, Gary Williams, MBBS, FCICM ${ }^{5}$, Lucy Chai See Lum, MBBS, MRCP ${ }^{6}$, Tavey Dorofaeff, MbChb $^{7}$, Pablo Cruces, MD ${ }^{8}$, Jenny L. Bush, RN, BSN ${ }^{9}$, Luke Keele, PhD ${ }^{10}$, Vinay M. Nadkarni, MD, $\mathbf{M S}^{9}$, Neal J. Thomas, MD, MSc ${ }^{11}$, Julie C. Fitzgerald, MD, PhD ${ }^{9}$, and Scott L. Weiss, MD, MSCE ${ }^{9}$ for the Sepsis PRevalence, OUtcomes, and Therapies Study Investigators and Pediatric Acute Lung Injury and Sepsis Investigators Network

${ }^{1}$ Division of Critical Care Medicine, Department of Pediatrics, Yale University School of Medicine, New Haven, CT ${ }^{2}$ Division of Pediatric Critical Care, Departments of Pediatrics and Anesthesiology Critical Care Medicine, Children's Hospital Los Angeles, University of Southern California Keck School of Medicine, Los Angeles, CA ${ }^{3}$ Department of Critical Care and Bioethics, Great Ormond Street Hospital NHS Trust, London, United Kingdom ${ }^{4}$ Department of Pediatrics, Royal Hospital for Children, Glasgow, Scotland ${ }^{5}$ Intensive Care Department, Sydney Children's Hospital, Randwick, NSW, Australia ${ }^{6}$ Department of Pediatrics, University of Malaya Medical Center, Kuala Lumpur, Malaysia ${ }^{7}$ Paediatric Intensive Care Unit, Division of Paediatric Critical Care, Department of Pediatrics, Lady Cilento Children's Hospital, Brisbane, QLD, Australia ${ }^{8}$ Department of Pediatric Intensive Care, Hospital El Carmen de Maipu, Universidad Andres Bello, Santiago, Chile ${ }^{9}$ Division of Critical Care Medicine, Department of Anesthesia and Critical Care, The Children's Hospital of Philadelphia, University of Pennsylvania Perelman School of Medicine, Philadelphia, PA ${ }^{10}$ Department of Political Science, Penn State University, University Park, PA

${ }^{11}$ Division of Pediatric Critical Care Medicine, Department of Pediatrics, Penn State Hershey Children's Hospital, Penn State University College of Medicine, Hershey, PA

\section{Abstract}

Objectives-Pediatric severe sepsis remains a significant global health problem without new therapies despite many multicenter clinical trials. We compared children managed with severe sepsis in European and U.S. PICUs to identify geographic variation, which may improve the design of future international studies.

Design-We conducted a secondary analysis of the Sepsis PRevalence, OUtcomes, and Therapies study. Data about PICU characteristics, patient demographics, therapies, and outcomes were compared. Multivariable regression models were used to determine adjusted differences in morbidity and mortality.

Setting一European and U.S. PICUs.

Supplemental digital content is available for this article. Direct URL citations appear in the printed text and are provided in the HTML and PDF versions of this article on the journal's website (http://journals.lww.com/pccmjournal)

For information regarding this article,: john.giuliano@yale.edu 
Patients-Children with severe sepsis managed in European and U.S. PICUs enrolled in the Sepsis PRevalence, OUtcomes, and Therapies study.

Interventions-None.

Measurements and Main Results-European PICUs had fewer beds (median, 11 vs 24; $p<$ 0.001 ). European patients were younger (median, 1 vs $6 \mathrm{yr} ; p<0.001$ ), had higher severity of illness (median Pediatric Index of Mortality $-3,5.0$ vs 3.8; $p=0.02$ ), and were more often admitted from the ward (37\% vs $24 \%$ ). Invasive mechanical ventilation, central venous access, and vasoactive infusions were used more frequently in European patients ( $85 \%$ vs $68 \%, p=0.002$; $91 \%$ vs $82 \%, p=0.05$; and $71 \%$ vs $50 \% ; p<0.001$, respectively). Raw morbidity and mortality outcomes were worse for European compared with U.S. patients, but after adjusting for patient characteristics, there were no significant differences in mortality, multiple organ dysfunction, disability at discharge, length of stay, or ventilator/vasoactive-free days.

Conclusions-Children with severe sepsis admitted to European PICUs have higher severity of illness, are more likely to be admitted from hospital wards, and receive more intensive care therapies than in the United States. The lack of significant differences in morbidity and mortality after adjusting for patient characteristics suggests that the approach to care between regions, perhaps related to PICU bed availability, needs to be considered in the design of future international clinical trials in pediatric severe sepsis.

\section{Keywords}

children; Europe; management; outcome; pediatric intensive care unit; shock

Pediatric severe sepsis remains a significant global health problem (1-6). In 2013, an estimated 6.3 million children died worldwide with 52\% resulting from infections (7). Identification of novel therapies will require a new phase of multicenter international clinical trials (8). However, substantial variability exists in pediatric infection mortality reported across geographic regions $(7,9)$. These differences may stem from several factors, including differences in the approach to healthcare delivery and availability of intensive care resources. Understanding systematic differences in patient characteristics, approach to therapy, and rate of adverse outcomes is necessary to ensure that existing regional variation is appropriately accounted for in the design of future multicenter international clinical trials for pediatric severe sepsis.

Data from the Surviving Sepsis Campaign have previously demonstrated a higher unadjusted mortality in adults with severe sepsis treated in European ICUs compared with the U.S. ICUs but noted that this difference disappeared after adjusting for admission source and illness severity. These results raise concerns that variability in the approach to critical care between regions, particularly with regard to ICU bed availability, may affect outcomes (10). Although such differences can modify the impact of a novel therapy if implemented based on the need for ICU admission, similar regional comparisons have not been made for children with severe sepsis treated in PICUs.

The Sepsis PRevalence, OUtcomes, and Therapies (SPROUT) study recently reported the prevalence, therapies, and outcomes of pediatric severe sepsis across PICUs worldwide. 
SPROUT investigators (Appendix 1) screened nearly 7,000 PICU patients in 128 sites across 26 countries for severe sepsis using pediatric sepsis consensus criteria (11). The largest number of sites and enrolled patients originated from Europe and the United States. For the current analysis, we used data from the SPROUT study to compare PICU characteristics, patient demographics, therapies, and outcomes for children with severe sepsis treated in Europe versus the United States. We hypothesized that disparities in outcomes for children with severe sepsis managed in Europe versus the United States would be largely explained by differences in patient characteristics.

\section{MATERIALS AND METHODS}

SPROUT was a prospective, cross-sectional point prevalence study with data collection performed on five study days spaced over 1 year from June 2013 to June 2014 (11). Followup data about outcomes were also collected. Sites were recruited by open invitation through established research networks, and participation was voluntary. Ethics approval was obtained at all sites with waiver of informed consent granted at all but three sites (two in Europe and one in the United States) at which written consent was required for data collection. Although the details of the SPROUT study methodology and primary results have been previously published $(11,12)$, the data in this study are limited to novel comparative analyses between Europe and the United States not previously reported.

All patients 18 years old being treated in a participating PICU at 9:00 AM local time on each study day were screened for severe sepsis using a standardized form with the "2005 International Pediatric Sepsis Consensus Conference" criteria: 1) at least two systemic inflammatory response syndrome criteria, 2) confirmed or suspected invasive infection, and 3) cardiovascular dysfunction, acute respiratory distress syndrome, or at least two organ dysfunctions (13). The subset of patients with septic shock defined by cardiovascular dysfunction was included within the spectrum of severe sepsis. Only clinical data available within the 24 hours preceding the 9:00 AM study day time were considered for screening, yielding a study cohort with active severe sepsis. Patients who were 18 years old or older, corrected gestational age less than 42 weeks, or who had surgery involving cardiopulmonary bypass in the preceding 5 days were excluded. Only patients enrolled with severe sepsis from a PICU in either Europe or the United States were included in the current analysis.

Data were collected about PICU characteristics and patient demographics, treatment, and outcomes within a 48-hour window around the study day (9:00 AM before to 9:00 AM after the study day). For severity of illness, the Pediatric Index of Mortality-3 (PIM3) score (14) was calculated at PICU admission and the Pediatric Logistic Organ Dysfunction score (15) was calculated on the study day. For all enrolled patients, the first-day meeting criteria for severe sepsis was determined. Multiple organ dysfunction syndrome (MODS) was defined as two or more concurrent organ system dysfunctions, and new or progressive MODS (NPMODS) was determined by following patients for seven consecutive days after severe sepsis recognition (16). Each patient was followed up for 90 days or until discharge from the hospital to determine mortality and functional disability outcomes. The Pediatric Overall Performance Category (POPC) ordinal scale (1 [normal] to 6 [death]) was used to assess new functional disability or change from baseline for all hospital survivors (17). Hospital- 
acquired infections (HAI) were those infections that occurred more than 48 hours following hospital admission.

The primary outcome of comparison was all-cause hospital mortality censored at 90 days from the study day or hospital discharge. Secondary outcomes included PICU mortality, PICU and hospital length of stay (LOS), vasoactive- and ventilator-free days from the day of severe sepsis recognition through day 28, NPMODS, and change in functional disability status (POPC). A composite outcome of death or moderate-severe disability worsened from baseline was also determined.

\section{Statistical Analysis}

Data were analyzed using STATA (version 12.1; StataCorp LP, College Station, TX). Categorical results are presented as proportions and analyzed using the Fisher exact or chisquare tests. Continuous data are presented as medians with interquartile range (IQR) and analyzed using the Wilcoxon signed rank test. Variables that differed between European and U.S. patients were tested as possible confounders in the association of geographic region with outcomes using multivariable regression models. We defined a confounder as a covariate that changed the odds ratio (OR) between region and the outcome by at least $10 \%$. The following covariates were tested for confounding: age, source of PICU admission, comorbid conditions, PIM3, MODS at sepsis recognition, site of infection, any bacteremia, and hospital- versus community-acquired infection. Although none of these variables met our a priori criteria for confounding, based on the strength of association with region in bivariate analyses, we used age, source of PICU admission, HAI, PIM3, and bacteremia in final multivariable models and present adjusted OR (aOR) with the 95\% CIs. We used logistic regression to test the association of region with outcomes for categorical outcomes and negative binomial regression for count outcomes (e.g., LOS and vasoactive-free days). Collinearity across covariates included in the final regression models was ruled out by ensuring the variance inflation factor was less than two for each variable.

Because U.S. sites included a larger number of older patients (leading to a substantial age discrepancy between groups as we note above), we included a control for age in the multivariate models. To further control for age, we also performed a supplemental analysis that assessed outcome differences independent of age by matching all European patients by age to a subsample of U.S. patients to better remove any residual confounding effect of age on outcome. We then reestimated the multivariate models using this matched dataset. In addition, because PIM3 uses systolic blood pressure uncorrected for age as part of the risk prediction, we tested whether observed regional differences in severity of illness reflected by PIM3 were attributable to the younger age of European patients by assessing both the correlation between age and PIM3 using Spearman correlation and comparing PIM3 in patients 1 year old or older versus less than 1 year old. We also attempted to account for siteto-site variation but given the low number of patients enrolled at each site, including a fixed effect for each site overfit the data and resulted in an incidental parameters problem. As an alternative, we estimated a mixed-effects logistic regression model, allowing for a random intercept by site. However, the inclusion of the random effect was not statistically significant, suggesting that baseline mortality did not vary significantly by site such that 
estimates from the mixed-effects logistic regression model were nearly identical to those from standard logistic regression. Therefore, for simplicity, we present only the results from standard regression models.

\section{RESULTS}

Centers in Europe and the U.S. screened a total of 5,639 PICU patients with 413 meeting criteria for severe sepsis. Two patients declined consent for data collection (both from the United States), leaving 411 patients in the final cohort. Europe included 39 sites with 86 patients (21\%), and the United States included 53 sites with 325 patients (79\%). Sites in Europe were less likely to be free-standing children's hospitals than those in the United States $(38 \%$ vs $51 \% ; p=0.001)$. In addition, there was a lower median number of PICU beds (11 [IQR, 8-15] vs 24 [IQR, 19-32]; $p<0.001)$ and annual PICU admissions (500 [IQR, 300-740] vs 1,300 [IQR, 850-1,800]; $p<0.001$ ) in Europe versus U.S. sites. The European and U.S. sites participating in the SPROUT study and included in this analysis are listed in Supplemental Table 1 (Supplemental Digital Content 1, http://links.lww.com/PCC/ A251).

The point prevalence of severe sepsis was $6.2 \%$ (95\% CI, 5.0-7.6) in European sites and 7.7\% (95\% CI, 6.9-8.5) in U.S. sites $(p=0.06)$. Table 1 shows patient characteristics by region. European patients were younger ( $1 \mathrm{yr}$ [IQR, 0.4-7 yr] vs 6 yr [IQR, 1-13 yr]; $p<$ 0.001 ) and had higher PIM3 scores (5.0 [IQR, 2.3-9.8] vs 3.8 [IQR, 1.6-7.9]; $p=0.02$ ) and higher proportions of MODS at sepsis recognition (73\% vs 51\%; $p<0.001)$. There was no correlation between age and PIM3 (Spearman $\rho, 0.02 ; p=0.67$ ) and median PIM3 did not differ by age 1 year or less (4.4 [IQR, 1.7-9.2] and > 1 yr [3.9 (IQR, 1.7-8.5)]; $p=0.40)$. The majority of patients from both regions had one or more comorbid conditions prior to admission (Europe: $88 \%$ vs United States: $86 \% ; p=0.60$ ). However, the types of comorbid conditions differed, with cardiovascular conditions predominating in Europe and respiratory, gastrointestinal, and neuromuscular conditions more common in the United States. More patients in Europe than the Unites States were admitted to PICUs from general hospital wards (37\% vs $24 \%$ ) rather than emergency departments (EDs) (8\% vs $35 \%)$.

The most common site of infection was respiratory in both regions but more patients in Europe had bacteremia than in the United States (32\% vs 19\%; $p=0.01$; Table 2). Grampositive bacteria were more commonly identified in European patients, whereas viruses were more often identified in U.S. patients. European patients had a higher proportion of HAIs ( $35 \%$ vs $25 \% ; p=0.004)$.

Therapies used in the 48-hour window around the SPROUT study days are presented in Table 3. The majority of patients in both regions had respiratory failure and were being actively mechanically ventilated within the time window of data collection. However, invasive mechanical ventilation was more common in Europe compared with U.S. patients ( $85 \%$ vs $68 \% ; p=0.002$ ) as were vasoactive infusions ( $71 \%$ vs $50 \% ; p<0.001)$. Dopamine was used more frequently in Europe ( $43 \%$ vs $25 \% ; p=0.02$ ), but epinephrine, norepinephrine, vasopressin, phenylephrine, dobutamine, and milrinone were used similarly between the two regions (all $p>0.05$ ). European patients were more likely to receive enteral 
nutrition (64\% vs 50\%; $p=0.03$ ), as well as blood products, synthetic colloid, and renal replacement therapies. Overall, central venous catheters were used slightly more often in European patients $(91 \%$ vs $82 \%$; $p=0.05)$, with peripherally inserted central catheters more common in the United States.

Unadjusted hospital and PICU mortality was higher in European compared with U.S. PICUs ( $30 \%$ vs $22 \%$ and $29 \%$ vs $22 \%$, respectively) although these differences did not reach statistical significance (Table 4). European patients had fewer vaso-active-free days, ventilator-free days, and longer hospital LOS than U.S. patients. NPMODS and mild and moderate disability at hospital discharge were not different between regions, and although the composite outcome of death or moderate disability trended higher in European patients, this did not reach statistical significance. After adjusting for age, source of PICU admission, HAI, PIM3, and bacteremia, there were no significant differences between European and U.S. patients for hospital mortality (aOR, 1.25; 95\% CI, 0.69-2.28; $p=0.46$ ), NPMODS (aOR, 0.68; 95\% CI, 0.39-1.19; $p=0.18$ ), or death/moderate disability (aOR, 1.02; 95\% CI, $0.59-1.75 ; p=0.95)$. Similarly, after adjusting for covariates, there were no longer significant differences in vasoactive-free days $(\beta,-0.17 ; 95 \% \mathrm{CI},-0.40$ to $0.06 ; p=0.14)$ or hospital $\operatorname{LOS}(\beta, 0.003 ; 95 \% \mathrm{CI},-0.21$ to $0.22 ; p=0.98)$ between regions. The supplementary analysis using age-matched patients further demonstrated no differences in outcomes between Europe and the United States (data not shown).

\section{DISCUSSION}

Using data from the recently completed SPROUT study, we compared pediatric patients treated for severe sepsis in European versus U.S. PICUs. European patients were more frequently admitted from hospital wards, were younger, had a higher severity of illness, had more HAIs, and received more intensive care therapies than patients in the United States. However, after adjusting for these factors, there were no significant differences in morbidity and mortality between regions. The lack of adjusted outcome differences raises concern that there may be a differential approach to care for hospitalized children with severe sepsis between regions, such that patients admitted to European PICUs are at a different phase of their septic illness than in the United States. Such differences in patient characteristics and care models need to be considered in the design of future international clinical trials in pediatric severe sepsis. In particular, it may be more appropriate to use consensus criteria to establish study eligibility irrespective of hospital location or PICU admission, especially if a study intervention is best delivered at initial sepsis recognition.

Our results concur with a recent study investigating severe sepsis in adult patients using the large voluntary Surviving Sepsis Campaign database. Paralleling our study, adult European ICUs possessed fewer beds but had more severely ill patients and admitted more patients from the hospital ward when compared with the U.S. ICUs. After accounting for differences in illness severity and source of ICU admission, raw differences in outcomes disappeared between European and U.S. adults with severe sepsis leading the authors to question the effect of regional differences in the approach to critical care, including ICU bed availability (10). In our study, we similarly found that European PICUs possessed fewer beds and admitted more severely ill patients who more often came from hospital wards rather than the 
ED. In addition, outcomes did not differ significantly after adjustment for baseline differences in illness severity for children with severe sepsis treated in European and U.S. PICUs.

The difference in severity of illness for pediatric patients with severe sepsis at admission to European versus U.S. PICUs is a notable finding. Admission PIM3 and the proportion with MODS at severe sepsis recognition were significantly higher in the European cohort of patients. Although PIM3 uses systolic blood pressure unadjusted for age as part of the risk prediction, there was no indication that the higher PIM3 distribution in European patients was explained by the overall lower age. Further reflecting the higher severity of illness, European patients more commonly received intensive care therapies than their U.S. counterparts. For example, the European group used more vasoactive infusions and mechanical ventilation. Although such treatment differences between regions could reflect practice variability, it is likely that these therapeutic differences underlie the higher baseline severity of illness on entering European PICUs.

Similar to the previously published adult study from the Surviving Sepsis Campaign, the median number of PICU beds per site was lower in Europe and more patients were admitted with severe sepsis from the general ward than the ED when compared with sites in the United States. This suggests that children are less likely to be triaged to an intensive care setting at hospital admission in Europe, which, consequently, leads to a smaller number of patients with an overall higher severity of illness at PICU admission. This difference in the number of available PICU beds may be an important factor in our observed trend toward a mortality difference in the unadjusted analyses because our study design did not account for those patients with severe sepsis who recovered on the wards and were never admitted to the PICU. The complete lack of difference in outcomes between regions after adjusting for severity of illness and source of admission further suggests that outcome differences may be related to differing thresholds for PICU admission between regions. Unfortunately, the SPROUT study did not capture illness severity at hospital admission or hospital LOS prior to PICU admission in order to compare triage decisions prior to the PICU. Further investigation into the illness severity threshold triggering PICU admission in each region may help to identify an optimal balance of PICU to total hospital beds. Interestingly, the PICU to total hospital bed ratio may be more important than the absolute number of PICU beds alone. For example, adult ICU patients admitted to low ICU-to-hospital bed ratio institutions used significantly more intensive care therapies than those admitted to institutions with high ratios (18). Future studies should consider the characteristics of the population served and the ratio of ICU to total available hospital beds, perhaps even extending to regional availability of beds, to account for interhospital transfers rather than the number of intensive care beds alone.

Other differences noted between patients in Europe and the United States were patient age, site of infection, and causative organism. European patients tended to be younger, have more primary bloodstream infections, bacteremia with Gram-positive bacteria, and HAIs. The age difference reflects a bimodal age distribution in the United States compared with a more right-skewed distribution in Europe (Supplemental Fig. 1, Supplemental Digital Content 2, http://links.lww.com/PCC/A252; legend: Age by region. The distribution of age [yr] is 
shown separately for PICU patients with severe sepsis in the United States and Europe. U.S. patients exhibited a bimodal age distribution, whereas European patients exhibited a rightskewed distribution.). This may reflect regional differences in patient flow in which newborns may be preferentially admitted to neonatal ICUs in the United States, whereas older adolescents are more likely to be treated in adult ICUs in Europe. It is possible that the higher rate of HAIs seen in Europe is a function of being admitted from the general hospital wards rather than from EDs as is generally the case in the United States. One prior study examining hospital-acquired bloodstream infections in Europe and the United States reported similar age- and sex-adjusted incidences per 100,000 person-years, but these estimates included only small numbers of children and were not representative of all of Europe or the United States (19). Our findings support the need for further comparative studies to better understand infection control practices in Europe and the United States.

A recent report outlining a roadmap to improve sepsis research noted that participation of additional clinical sites will be necessary to account for a declining case fatality rate and to better address patient heterogeneity in sepsis (8). For pediatrics, this will increasingly require international cooperation. Based on the findings of our study, we suggest that future international clinical trials should use physiologic and laboratory criteria for severe sepsis to establish study eligibility without the requirement for PICU admission. This approach would be most important for testing therapies that need to be administered near sepsis recognition, such as antibiotic timing, choice of fluid resuscitation, early blood transfusions, and initial vasoactive infusion selection. Many of these therapies are likely to begin and evolve outside of the PICU setting, particularly in regions with few available PICU beds. In addition, epidemiologic studies comparing incidence and outcomes across geographic regions need to account for the apparent differential threshold for PICU admission to better understand how patient care is transferred between units within different hospitals. Although we endorse this approach, the precise physiologic and laboratory criteria used to identify pediatric sepsis and the optimal approach to ensure early and complete recognition remain important challenges (8).

This study has several important limitations. First, given the cross-sectional nature of data collection limited to a brief window of time, it is likely that the use of therapies over the entire course of illness was underestimated. Second, although most organ dysfunction in pediatric sepsis occurs in the first 7 days, some cases of NPMODS may have been missed by only measuring organ dysfunction for seven instead of 28 days following sepsis recognition (16). Third, we were not able to compare illness severity at hospital admission, duration of hospital LOS prior to PICU admission, therapies received at PICU admission, or the quality of the initial resuscitation for septic shock. It is possible that some of the differences observed in use of intensive therapies may reflect regional differences in sepsis recognition and early management. Similarly, we cannot determine if practice variation in viral testing contributed to the higher proportion of viral sepsis noted in the United States. Fourth, as this was a secondary analysis of an existing dataset, power may have been insufficient to detect true differences in outcome. This limitation is reflected in the width of our 95\% CIs, which suggests that the true OR for hospital mortality may be as high as 2.28 for European versus U.S. patients. In addition, this study was limited to PICU patients and thus does not account for mortality that occurred outside of this setting. A recent study from the United Kingdom 
suggested that early deaths in pediatric sepsis may be important to consider (20). Finally, we have compared the care practices and outcomes for patients from one country (United States) with those of many countries throughout Europe. Other than the United Kingdom and Spain, each European country contributed data from only a few sites. Consequently, the generalizability of our findings to any single European country is limited. Similarly, there were not sufficient numbers of sites from other geographic regions in the SPROUT study to facilitate broader global comparisons. Still, the finding that patient characteristics and therapies differed between regions and accounted for much of the raw differences in outcomes supports the need to account for regional variability in multicenter international studies.

\section{CONCLUSIONS}

Despite European PICU patients with severe sepsis having a younger age, higher severity of illness, more HAIs, and more frequent need for intensive care therapies than those in the United States, there were no significant differences in adjusted estimates of morbidity and mortality between regions. Our findings suggest that the approach to care between regions, perhaps related to PICU bed availability, needs to be considered in the design of future international clinical trials in pediatric severe sepsis. Patients meeting consensus definitions rather than PICU admission may be a more appropriate criterion of study eligibility.

\section{Supplementary Material}

Refer to Web version on PubMed Central for supplementary material.

\section{Acknowledgments}

We acknowledge the contributions of all the participating Sepsis PRevalence, OUtcomes, and Therapies investigators.

Supported, in part, by the Endowed Chair, Department of Anesthesia and Critical Care, University of Pennsylvania Perelman School of Medicine and the Center for Pediatric Clinical Effectiveness at The Children's Hospital of Philadelphia. Financial support for data collection in all U.K. centers was provided by the U.K. National Institute of Health (NIHR) Clinical Research Network and in Southampton by the Southampton NIHR Welcome Trust Clinical Research Facility. None of the funders participated in the design and conduct of the study; collection management, analysis and interpretation of the data; or preparation, review, or approval of the article.

Dr. Giuliano Jr received support for article research from the National Institutes of Health (NIH), Wellcome Trust/ COAF, and Research Councils UK (RCUK). Dr. Brierley received funding from Astellas. Dr. Keele's institution received funding from Picwell Corp and from the Department of Labor. Dr. Thomas served as a board member for Therabon (Advisory Board) and received support for lectures from CareFusion (Webinar). His institution received grant support from the FDA (R01). Dr. Fitzgerald's institution received funding from the Children's Hospital of Philadelphia Center for Pediatric Clinical Effectiveness. Dr. Weiss received funding from Thermo-Fisher Scientific (honoraria for lecture unrelated to this study topic). He was also supported by National Institute of Child Health and Human Development (K12HD047349 and NIGMS K23GM110496). His institution received funding from The Children's Hospital of Philadelphia Center for Pediatric Clinical Effectiveness. The remaining authors have disclosed that they do not have any potential conflicts of interest.

\section{References}

1. Hartman ME, Linde-Zwirble WT, Angus DC, et al. Trends in the epidemiology of pediatric severe sepsis*. Pediatr Crit Care Med. 2013; 14:686-693. [PubMed: 23897242] 
2. Jaramillo-Bustamante JC, Marin-Agudelo A, Fernandez-Laverde M, et al. Epidemiology of sepsis in pediatric intensive care units: First Colombian multicenter study. Pediatr Crit Care Med. 2012; 13:501-508. [PubMed: 22460772]

3. Watson RS, Carcillo JA. Scope and epidemiology of pediatric sepsis. Pediatr Crit Care Med. 2005; 6:S3-S5. [PubMed: 15857554]

4. Ganjoo S, Ahmad K, Qureshi UA, et al. Clinical epidemiology of SIRS and sepsis in newly admitted children. Indian J Pediatr. 2015; 82:698-702. [PubMed: 25429997]

5. Ruth A, McCracken CE, Fortenberry JD, et al. Pediatric severe sepsis: Current trends and outcomes from the Pediatric Health Information Systems database. Pediatr Crit Care Med. 2014; 15:828-838. [PubMed: 25226500]

6. Balamuth F, Weiss SL, Neuman MI, et al. Pediatric severe sepsis in U.S. children's hospitals. Pediatr Crit Care Med. 2014; 15:798-805. [PubMed: 25162514]

7. Liu L, Oza S, Hogan D, et al. Global, regional, and national causes of child mortality in 2000-13, with projections to inform post-2015 priorities: An updated systematic analysis. Lancet. 2015; 385:430-440. [PubMed: 25280870]

8. Cohen J, Vincent JL, Adhikari NK, et al. Sepsis: A roadmap for future research. Lancet Infect Dis. 2015; 15:581-614. [PubMed: 25932591]

9. Wang H, Liddell CA, Coates MM, et al. Global, regional, and national levels of neonatal, infant, and under-5 mortality during 1990-2013: A systematic analysis for the Global Burden of Disease Study 2013. Lancet. 2014; 384:957-979. [PubMed: 24797572]

10. Levy MM, Artigas A, Phillips GS, et al. Outcomes of the Surviving Sepsis Campaign in intensive care units in the USA and Europe: A prospective cohort study. Lancet Infect Dis. 2012; 12:919_ 924. [PubMed: 23103175]

11. Weiss SL, Fitzgerald JC, Pappachan J, et al. Sepsis Prevalence, Outcomes, and Therapies (SPROUT) Study Investigators and Pediatric Acute Lung Injury and Sepsis Investigators (PALISI) Network: Global epidemiology of pediatric severe sepsis: The sepsis prevalence, outcomes, and therapies study. Am J Respir Crit Care Med. 2015; 191:1147-1157. [PubMed: 25734408]

12. Weiss SL, Fitzgerald JC, Faustino EV, et al. Pediatric Acute Lung Injury and Sepsis Investigators Network and Australia and New Zealand Intensive Care Society Investigators: Understanding the global epidemiology of pediatric critical illness: The power, pitfalls, and practicalities of point prevalence studies. Pediatr Crit Care Med. 2014; 15:660-666. [PubMed: 24751790]

13. Goldstein B, Giroir B, Randolph A. International Consensus Conference on Pediatric Sepsis: International pediatric sepsis consensus conference: Definitions for sepsis and organ dysfunction in pediatrics. Pediatr Crit Care Med. 2005; 6:2-8. [PubMed: 15636651]

14. Straney L, Clements A, Parslow RC, et al. ANZICS Paediatric Study Group and the Paediatric Intensive Care Audit Network: Paediatric index of mortality 3: An updated model for predicting mortality in pediatric intensive care*. Pediatr Crit Care Med. 2013; 14:673-681. [PubMed: 23863821]

15. Leteurtre S, Martinot A, Duhamel A, et al. Validation of the paediatric logistic organ dysfunction (PELOD) score: Prospective, observational, multicentre study. Lancet. 2003; 362:192-197. [PubMed: 12885479]

16. Proulx F, Gauthier M, Nadeau D, et al. Timing and predictors of death in pediatric patients with multiple organ system failure. Crit Care Med. 1994; 22:1025-1031. [PubMed: 8205810]

17. Fiser DH. Assessing the outcome of pediatric intensive care. J Pediatr. 1992; 121:68-74. [PubMed: 1625096]

18. Sasabuchi Y, Yasunaga H, Matsui H, et al. The volume-outcome relationship in critically ill patients in relation to the ICU-to-hospital bed ratio. Crit Care Med. 2015; 43:1239-1245. [PubMed: 25756414]

19. Goto M, Al-Hasan MN. Overall burden of bloodstream infection and nosocomial bloodstream infection in North America and Europe. Clin Microbiol Infect. 2013; 19:501-509. [PubMed: 23473333]

20. Cvetkovic M, Lutman D, Ramnarayan P, et al. Timing of death in children referred for intensive care with severe sepsis: Implications for interventional studies. Pediatr Crit Care Med. 2015; 16:410-417. [PubMed: 25739013] 


\section{APPENDIX 1. SPROUT STUDY INVESTIGATORS}

North America: Canada: P. Fontela (Montreal Children's Hospital-McGill), M. Tucci, M.

Dumistrascu (Sainte Justine Hospital), P. Skippen, G. Krahn (BC Children's Hospital);

Puerto Rico: E. Bezares (Hospital Cardiovascular de Puerto Rico y el Caribe), G. Puig, A. Puig-Ramos (San Jorge Children's Hospital), R. Garcia, M. Villar (University Pediatric

Hospital); United States: M. Bigham, T. Polanski, S. Latifi, D. Giebner, H. Anthony (Akron Children's Hospital), J. Hume, A. Galster, L. Linnerud (Amplatz Children's Hospital), R. Sanders, G. Hefley (Arkansas Children's Hospital), K. Madden (Boston Children's Hospital), A. Thompson, S. Shein (Children's Hospital of Pittsburgh), S. Gertz (Children's Hospital-Hackensack), Y. Han, T. Williams, A. Hughes-Schalk (Children's Mercy Hospital), H. Chandler (Children's Healthcare of Atlanta), A. Orioles, E. Zielinski, A. Doucette (Children's Hospital in Minnesota), A. Orioles, E. Zielinski, A. Doucette (Children's Hospital St. Paul), C. Zebuhr, T. Wilson (Children's Hospital Colorado), C. Dimitriades, J. Ascani, S. Layburn, S. Valley (Children's Hospital New Orleans), B. Markowitz, J. Terry, R. Morzov (Children's Hospital of Los Angeles), A. Mcinnes (Children's Hospital of Monmouth), J. McArthur, K. Woods, K. Murkowski (Children's Hospital of Wisconsin), M. Spaeder, M. Sharron (Children's National Medical Center), D. Wheeler, E. Beckman, E. Frank, K. Howard (Cincinnati Children's Medical Center), C. Carroll (Connecticut Children's), S. Nett, D. Jarvis (Dartmouth Hitchcock), V. Patel (Dayton Children's Hospital), R. Higgerson, L. Christie (Dell Children's Medical Center), K. Typpo, J. Deschenes (Diamond Children's Hospital), A. Kirby (Doernbecher Children's Hospital), T. Uhl, K. Rehder, I. Cheifetz, S. Wrenn (Duke Children's Hospital), K. Kypuros (El Paso Children's Hospital), K. Ackerman (Golisano Children's Hospital), F. Maffei, G. Bloomquist (Janet Weis/Geisinger), N. Rizkalla (Johns Hopkins), D. Kimura, S. Shah, C. Tigges (Le Bonheur Children's Hospital), F. Su, C. Barlow (Lucile Pack-ard Children's Hospital), K. Michelson, K. Wolfe, D. Goodman, L. Campbell, L. Sorce (Lurie Children's Hospital of Chicago), K. Bysani, T. Monjure (Medical City Children's-Dallas), M. Evans (Medical University of South Carolina), B. Totapally, M. Chegondi, C. Rodriguez (Miami Children's Hospital), J. Frazier, L. Steele (Nationwide Children's Hospital), S. Viteri, A. Costarino (Nemours/Alfred I. duPont Children's Hospital), N. Thomas, D. Spear (Penn State Hershey Medical Center), E. Hirshberg, J. Lilley (Primary Children's Medical Center), C. Rowan, C. Rider (Riley Hospital for Children), J. Kane (Rush Children's Hospital), J. Zimmerman, C. Greeley (Seattle Children's Hospital), J. Lin, R. Jacobs (St. Louis Children's Hospital), M. Parker, K. Culver (Stony Brook University), L. Loftis, N. Jaimon, M. Goldsworthy (Texas Children's Hospital), J. Fitzgerald, S. Weiss, V. Nadkarni, J. Bush, M. Diliberto (The Children's Hospital of Philadelphia), C. Alen, M. Gessouroun (Oklahoma University Medical Center), A. Sapru, T. Lang, M. Alkhouli (University of California San Francisco), S. Kamath, D. Friel, J. Daufeldt (University of Iowa), D. Hsing, C. Carlo, S. Pon (Weill Cornell Medical Center), J. Scimeme, A. Shaheen (Wolfson Children's Hospital), A. Hassinger, H. Qiao (Women and Children's Hospital of Buffalo), J. Giuliano, J. Tala (Yale Children's Hospital). South America: Argentina: D. Vinciguerra, A. Fernandez (Hospital Durand); Colombia: R. Carrero (Clínica Infantil Colsubsidio), P. Hoyos (Hospital de San Jose), J. Jaramillo, A. Posada (Hospital General de Medellín), L. Izquiierdo (Hospital Military Central), B.E. Piñeres Olave, J. Donado (Pablo Tobón Uribe); Chile: R. Dalmazzo, 
S. Rendich (Clínica Las Condes), L. Palma, M. Lapadula (Clínica Santa María), C. Acuna (Hospital Luis Calvo Mackenna), P. Cruces (Hospital Padre Hurtado) Europe: Belgium: S. Clement De Clety, M. Dujardin, C. Berghe, S. Renard (St. Luc University Hospital); Czech

Republic: J. Zurek (Masaryk University); Germany: H. Steinherr (Klinikum Augsburg); Greece: K. Mougkou (Aghia Sophia Children's Hospital), E. Critselis, K. Mougkou (P. \& A. Kyriakou Children's Hospital); Italy: M. Di Nardo, S. Picardo, F. Tortora (Bambino Gesu Area Rossa); E. Rossetti (Bambino Gesu Children's Hospital); T. Fragasso, P. Cogo, R. Netto (Bambino Gesu Pediatrico); Lithuania: A. Dagys, V. Gurskis, R. Kevalas (Lithuanian University of Health Sciences); Netherlands: C. Neeleman, J. Lemson, C. Luijten (Radboud University Medical Centre); Poland: K. Wojciech, I. Pagowska-Klimek (Polish Mother Memorial Hospital), M. Szczepanska, J. Karpe (Szyszko Śląskiego University); Portugal: P. Nunes, H. Almeida (Hospital Prof Dr. Fernando Fonseca), J. Rios, M. Vieira (Centrol Hospitalar Lisboa Norte); Spain: P. Revilla (Children's Hospital Miguel Servet), J. Urbano, J. Lopez-Herce, A. Bustinza (Hospital General Universitario Gregorio Marañón), A. Cuesta, S. Hofheinz (Hospital 12 de Octubre), A. Rodriguez-Nunez (Hospital Clínico Universitario), S. Sanagustin, E. Gonzalez (Hospital de la Sant Creu Sant Pau), M. Riaza, R. Piaya (Hospital Universitario Madrid), P. Soler (Hospital Carlos Haya Materno Infantil), E. Esteban (Hospital Sant Joan de Déu), J. Laraudogoitia, C. Monge (Hospital Universitario Donostia), V. Herrera, J. Granados (Hospital Universitario Salamanca), C. Gonzalez (Hospital Virgen de la Arrixaca); Turkey: T. Koroglu, E. Ozcelik (Dokuz Eylul University); United Kingdom: P. Baines (Alder Hey Children's Hospital), A. Plunkett (Birmingham Children's Hospital), P. Davis, S. George (Bristol Royal Hospital for Children), S. Tibby, J. Harris (Evelina Children's Hospital), R. Agbeko, R. Lampitt (Great North Children's Hospital-Newcastle), J. Brierley, M. Peters, A. Jones, T. Dominguez, T. Thiruchelvam, (Great Ormond Street), A. Deep, L. Ridley, W. Bowen (King's College Hospital), R. Levin, I. Macleod (Royal Hospital for Sick Children), M. Gray, N. Hemat (St George's Hospital), J. Alexander, S. Ali (University Hospital of North Staffordshire NHS Trust), J. Pappachan, J. McCorkell (University Hospital Southampton NHS Foundation Trust), P. Fortune, M. MacDonald, P. Hudnott (Royal Manchester Children's Hospital). Asia: China: Q. Suyun (Beijing Children's Hospital); India: S. Singhi, K. Nallasamy (Advanced Pediatrics), R. Lodha (All India Institute); Japan: N. Shime, Y. Tabata (Kyoto Prefectural), O. Saito, T. Ikeyama (Tokyo Metropolitan), T. Kawasaki (Shizuoka Children's Hospital); Malaysia: L. Lum, A. Abidin, S. Kee (University Malaya Medical Center), S. Tang, R. Jalil (Kebangsaan Malaysia Medical Center); Singapore: Y. Guan, L. Yao (KK Women's and Children's Hospital), K. Lin, J. Ong (National University Hospital). Africa: South Africa: A. Salloo, L. Doedens, L. Mathivha (Chris Hani Baragwanath), G. Reubenson, S. Moaisi (Rahima Moosa), A. Pentz, R. Green (Steve Biko Academic Hospital). Australia: A. Schibler, A. Fernandez (Mater Children's Hospital), S. Erickson (Princess Margaret Hospital), J. McEneiry, D. Long, T. Dorofaeff, M. Coulthard (Royal Children's Hospital Brisbane), J. Millar, C. Delzoppo (Royal Children's Melbourne), G. Williams, M. Morritt (Sydney Children's Hospital), N. Watts, M. Morritt (Children's Hospital Westmead). New Zealand: J. Beca, C. Sherring, T. Bushell (Starship Children's Hospital) 


\section{TABLE 1}

\section{Patient Characteristics by Region}

\begin{tabular}{|c|c|c|c|}
\hline Variable & Europe & United States & $p$ \\
\hline Patients, $n$ & 86 & 325 & \\
\hline Age $(y r)$ & $1(0.4-7)$ & $6(1-13)$ & $<0.001$ \\
\hline Sex (male) & $48(56)$ & $169(52)$ & 0.55 \\
\hline \multicolumn{4}{|l|}{ Race, $n(\%)$} \\
\hline White & $64(74)$ & $168(52)$ & $<0.001$ \\
\hline Black & $4(5)$ & $61(19)$ & \\
\hline Asian & $10(12)$ & $10(3)$ & \\
\hline Other/unknown & $8(9)$ & $88(27)$ & \\
\hline \multicolumn{4}{|l|}{ Source of PICU admission, $n(\%)$} \\
\hline Emergency department & $7(8)$ & $113(35)$ & $<0.001$ \\
\hline Hospital ward & $32(37)$ & $79(24)$ & \\
\hline Operating room & $13(15)$ & $24(7)$ & \\
\hline Other hospital & $31(36)$ & $92(28)$ & \\
\hline Other & $3(3)$ & $17(5)$ & \\
\hline Previously healthy, $n(\%)$ & $10(12)$ & $46(14)$ & 0.60 \\
\hline \multicolumn{4}{|l|}{ Comorbid conditions, ${ }^{a} n(\%)$} \\
\hline Respiratory & $22(26)$ & $129(40)$ & 0.02 \\
\hline Gastrointestinal & $16(19)$ & $111(24)$ & 0.006 \\
\hline Cardiovascular & $33(38)$ & $82(25)$ & 0.02 \\
\hline Genetic & $15(17)$ & $88(27)$ & 0.07 \\
\hline Hematologic/immunologic & $19(22)$ & $76(23)$ & 0.89 \\
\hline Neuromuscular & $10(12)$ & $76(23)$ & 0.02 \\
\hline Neoplastic & $11(13)$ & $48(15)$ & 0.73 \\
\hline Prematurity & $12(14)$ & $56(17)$ & 0.52 \\
\hline Metabolic & $12(14)$ & $41(13)$ & 0.72 \\
\hline Renal & $5(6)$ & $42(13)$ & 0.09 \\
\hline Solid organ/stem cell transplant & $10(12)$ & $38(12)$ & 0.99 \\
\hline Pediatric Index of Mortality- $3 b$ & $5.0(2.3-9.8)$ & $3.8(1.6-7.9)$ & 0.02 \\
\hline Pediatric Logistic Organ Dysfunction $c$ & $11(2-20)$ & $11(2-12)$ & 0.49 \\
\hline Multiple organ dysfunction syndrome at sepsis recognition $d$ & $63(73)$ & $166(51)$ & $<0.001$ \\
\hline
\end{tabular}

${ }^{a}$ Categories do not add up to $100 \%$ because some patients had multiple comorbid conditions.

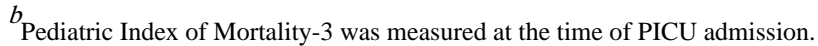




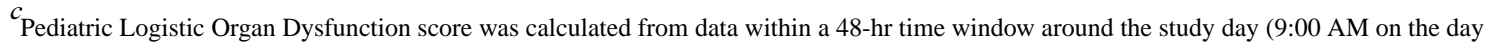
before to 9:00 AM on the day after the study day).

$d$ Multiple organ dysfunction syndrome based on criteria by Proulx et al [16].

Data presented as median (interquartile range) unless noted. 
TABLE 2

Site and Type of Infection Identified by Region

\begin{tabular}{lccc}
\hline Characteristic & Europe & United States & $p$ \\
\hline Primary site of infection & & & \\
Respiratory & $32(37)$ & $136(42)$ & 0.002 \\
Primary bloodstream & $26(30)$ & $48(15)$ & \\
Abdominal & $11(13)$ & $17(5)$ & \\
Central nervous system & $2(2)$ & $12(4)$ & \\
Genitourinary & $2(2)$ & $15(5)$ & \\
Skin & 0 & $8(2)$ & \\
Other & $3(3)$ & $18(6)$ & \\
Unknown & $10(12)$ & $70(22)$ & \\
Any bacteremia ${ }^{a}$ & $28(32)$ & $63(19)$ & 0.01 \\
\hline Microbiology $b$ & $9(10)$ & $79(24)$ & 0.005 \\
\hline Gram-positive bacteria & $31(36)$ & $77(24)$ & 0.03 \\
Gram-negative bacteria & $26(30)$ & $86(26)$ & 0.50 \\
Fungus & $12(15)$ & $43(13)$ & 0.72 \\
Virus & & & \\
No organism identified & $25(29)$ & $124(38)$ & 0.13 \\
\hline & & & \\
\hline & & & \\
\hline
\end{tabular}

${ }^{a}$ Patients with secondary bacteremia (e.g., pneumonia as primary site of infection with associated bacteremia).

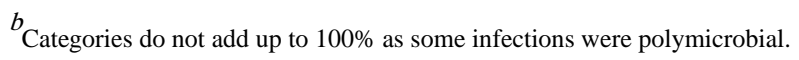

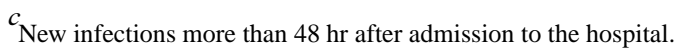

Data presented as $n(\%)$. 
TABLE 3

Regional Comparison of Therapies Used Within the 48-Hour Data Collection Window

\begin{tabular}{|c|c|c|c|}
\hline Therapy & Europe & United States & $p$ \\
\hline Invasive mechanical ventilation & $73(85)$ & $222(68)$ & 0.002 \\
\hline Vasoactive infusion $^{a}$ & $61(71)$ & $161(50)$ & $<0.001$ \\
\hline Synthetic colloid & $12(14)$ & $4(1)$ & $<0.001$ \\
\hline Albumin & $25(29)$ & $66(20)$ & 0.11 \\
\hline Blood products $b$ & $44(51)$ & $118(36)$ & 0.01 \\
\hline Corticosteroids & $31(36)$ & $152(47)$ & 0.09 \\
\hline Hydrocortisone $e^{c}$ & $19(61)$ & $81(53)$ & 0.44 \\
\hline Prednisone/methylprednisone ${ }^{c}$ & $9(29)$ & $53(35)$ & 0.68 \\
\hline Insulin $d$ & $9(10)$ & $41(13)$ & 0.71 \\
\hline Granulocyte/granulocyte-macrophage colony stimulating factor & $2(2)$ & $13(4)$ & 0.75 \\
\hline IV immunoglobulin & $6(7)$ & $17(5)$ & 0.60 \\
\hline Renal replacement therapy ${ }^{e}$ & $20(23)$ & $37(11)$ & 0.008 \\
\hline Plasma exchange & $1(<1)$ & $1(<1)$ & 0.38 \\
\hline Extracorporeal membrane oxygenation & $2(2)$ & $22(7)$ & 0.19 \\
\hline Nutrition, enteral & $55(64)$ & $163(50)$ & 0.03 \\
\hline Nutrition, parenteral & $40(47)$ & $140(43)$ & 0.63 \\
\hline \multicolumn{4}{|l|}{ Central venous catheter } \\
\hline Tunneled or implantable & $17(20)$ & $68(21)$ & 0.88 \\
\hline Nontunneled & $60(70)$ & $119(37)$ & $<0.001$ \\
\hline Peripherally inserted central catheter & $5(6)$ & $104(32)$ & $<0.001$ \\
\hline
\end{tabular}

Dopamine $>5 \mathrm{mg} / \mathrm{kg} / \mathrm{min}$, dobutamine $>5 \mathrm{mg} / \mathrm{kg} / \mathrm{min}$, or any dose of epinephrine, norepinephrine, vasopressin, phenylephrine, milrinone, levosimendan, or a vasodilator.

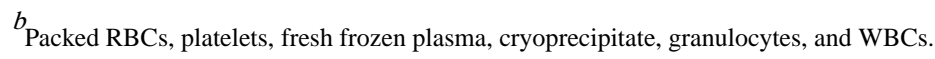

$c$ Denominator is the number of patients receiving any vasoactive infusion ( $n=152$ for United States; $n=31$ for Europe).

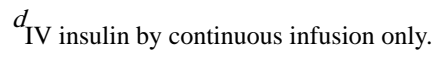

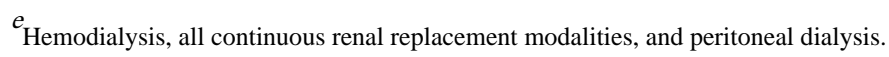

Data presented as $n(\%)$. 


\section{TABLE 4}

\section{Unadjusted Patient Outcomes by Region}

\begin{tabular}{llcc}
\hline Outcome Measure & Europe & United States & $p$ \\
\hline Hospital mortality & $26(30)$ & $73(22)$ & 0.16 \\
\hline PICU mortality & $25(29)$ & $70(22)$ & 0.15 \\
\hline Hospital LOS, median (IQR) & $31(19-73)$ & $24(12-56)$ & 0.007 \\
\hline PICU LOS, median (IQR) & $18(11-39)$ & $15(6-38)$ & 0.08 \\
\hline Vasoactive-free days, median (IQR) & $21(2-26)$ & $25(15-28)$ & $<0.001$ \\
\hline Ventilator-free days, median (IQR) & $15(0-22)$ & $17(0-26)$ & 0.08 \\
\hline New or progressive multiple organ dysfunction syndrome & $33(38)$ & $128(39)$ & 0.90 \\
\hline At least mild disability $b$ & $17(28)$ & $67(27)$ & 0.87 \\
\hline At least moderate disability $c$ & $11(18)$ & $48(19)$ & 0.99 \\
\hline Death or moderate disability $d^{c}$ & $37(43)$ & $121(37)$ & 0.38 \\
\hline
\end{tabular}

LOS = length of stay, IQR = interquartile range.

${ }^{a}$ New or progressive multiple organ dysfunction syndrome was considered starting the day after sepsis recognition.

${ }^{b}$ Any increase in Pediatric Overall Performance Category from baseline to hospital discharge in the 312 hospital survivors.

$c$ Discharge Pediatric Overall Performance Category $\geq 3$ and an increase of $\geq 1$ from baseline in the 312 hospital survivors.

${ }^{d}$ Death or at least moderate disability at discharge.

Data presented as $n(\%)$ unless noted. 